\title{
Experimental study of a series-connected two-evaporator refrigerating system with propane (R-290) as the refrigerant
}

\author{
Chao-Jen Li, Chin-Chia Su* \\ Department of Mechanical Engineering, National Taiwan University, No. 1, Sec. 4, Roosevelt Road, Taipei 106, \\ Taiwan, $R O C$
}

Received 18 November 2002; accepted 16 March 2003

\begin{abstract}
The performance of a refrigerating system with an environment-friendly refrigerant, propane (R-290) as the refrigerant, was experimentally studied. There were two evaporators connected in series within the system under study.

The results show that with both lengths of the two capillary tubes fixed, both the mass flow rate of the refrigerant and the suction pressure of the system increase with the condensing pressure. In addition, the cooling capacity of the high-temperature evaporator decreases, but that of the low-temperature evaporator increases. As the condensing pressure is fixed and the length of the capillary tube for the high-temperature evaporator is increased while that for the low-temperature evaporator is fixed, the cooling capacity of the high-temperature evaporator increases while that of the low-temperature evaporator decreases. On the other hand, as the capillary tube for the low-temperature evaporator is lengthened while that for the hightemperature evaporator is fixed, the variations in the cooling capacity of these two evaporators reverse. The enthalpy changes of the refrigerant within the evaporators are strongly affected by the length of the hightemperature capillary tube, while the evaporating pressures are influenced mainly by the length of the lowtemperature capillary tube.
\end{abstract}

(c) 2003 Elsevier Science Ltd. All rights reserved.

Keywords: Propane; R-290; Two-evaporator; Refrigerating system

\footnotetext{
${ }^{*}$ Corresponding author. Tel./fax: +886-2-2368-7352.

E-mail address: chinchiasu@ccms.ntu.edu.tw (C.-C. Su).
} 


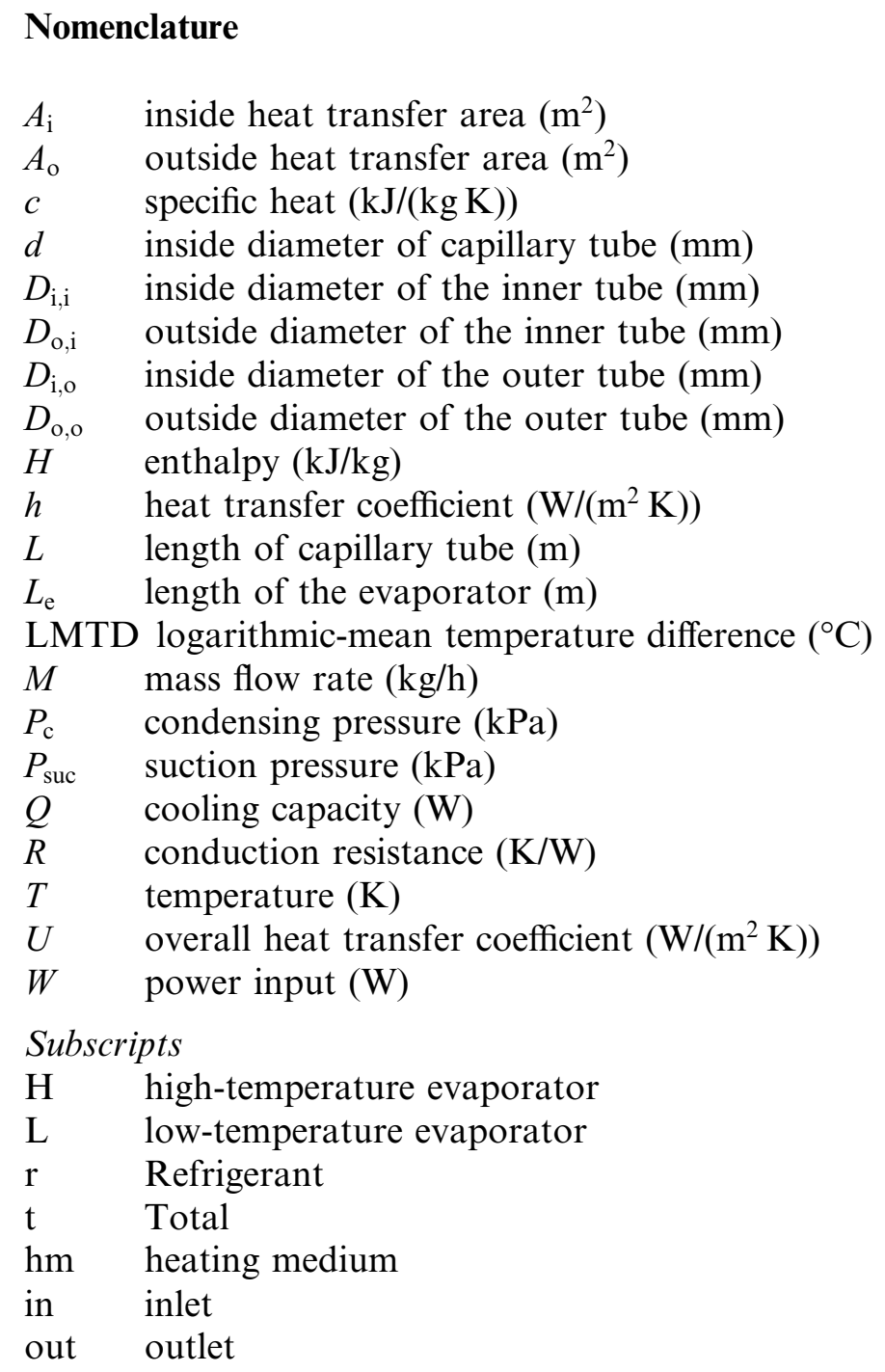

Subscripts

$\mathrm{H}$ high-temperature evaporator

L low-temperature evaporator

r Refrigerant

$\mathrm{t}$ Total

hm heating medium

in inlet

out outlet

\section{Introduction}

It is well known that the impacts of the refrigerant on the ozone depleting potential (ODP) and the global warming potential (GWP) are determined by the chlorine and fluorine, respectively, contained in the refrigerants. Refrigerants containing chlorine or fluorine, such as R-22, HFC134a, etc., should therefore be replaced by environment-friendly substances, containing neither chlorine nor fluorine, hydrocarbons (HCs) represent a zero ODP and an extremely low GWP solution. From technical and thermodynamic points of view, some HCs are good alternatives. The refrigerating properties of propane (R-290) are close to those of R-22. R-290 is, therefore, a 
potential alternative to R-22. In addition, the compatibility of R-290 with the conventional mineral oil used in R-22 systems is very good.

The performance of the refrigerating system with HCs as the refrigerant has been investigated (e.g. [1-5]). However, the investigation was concentrated on the conventional system with only one evaporator. A refrigerating system with two or more evaporators connected in series may give better performance than that with only one evaporator. From the thermodynamic viewpoint, decreasing the temperature difference between evaporation and condensation increases the energy efficiency. The advantage may be availed with two evaporators, in which the temperature difference for the high-temperature evaporator is smaller. For example, a two-evaporator refrigerating system charged with nonazeotropic refrigerant R22/R11 $(50 \% / 50 \%$ by weight) shows a power saving of $20 \%$ compared with that of R-12 with only one evaporator [6]. Similar results were obtained by Rose et al. [7]. The computer simulation of a two-evaporator refrigerating system charged with pure and mixed refrigerants, conducted by Jung and Radermacher [8], shows a significant increase in COP. The cycle proposed by Lorenz and Meutzner [6] was modified by Simmons et al. $[9,10]$ with some bypasses to the compressor. Again the experimental results showed that the energy saving potential is high with two evaporators in series.

It should be pointed out that although there are two evaporators in both the original cycle proposed by Lorenz and Meutzner [6] and the modified one [9,10], there is only one capillary tube in each system. Different evaporating temperatures are achieved through the nonazeotropic characteristics of the mixed refrigerants. With a single refrigerant, different evaporating temperatures can only be obtained by the use of multiple capillary tubes and evaporators. To this end, an experimental system was established to explore the performance of the two-evaporator refrigerating system with pure R-290 as the refrigerant.

\section{Experimentation}

Fig. 1 shows the schematic of the experimental system setup for specific purpose. The experimental apparatus can be divided into three subsystems: one refrigerant loop and two heatexchange fluid loops. The states of the working fluids at appropriate locations were monitored with T-type thermocouples and pressure gauges as shown.

As shown in Fig. 1, the refrigerant loop is composed of a reciprocating compressor (L51B562DBL B, Bristol), a condenser, a filter-dryer, a refrigerant flow meter, a sight glass, an electromagnetic valve, two capillary tubes, two evaporators, and some valves. A capillary tube is arranged before each evaporator.

The power input of the three-phase, $220 \mathrm{~V}$, reciprocating compressor is controlled by the frequency converter. The output of the converter can be adjusted between 0 and $120 \mathrm{~Hz}$, but the range for stable operation is from 40 to $80 \mathrm{~Hz}$. The lubricant of the compressor is a $150 \mathrm{SSU}$ mineral oil. The condenser is a finned-tube heat exchanger. The condensation of the refrigerant in the tube is effected by the cooling air, which is induced by the action of a fan inside the condenser. The airflow rate is controlled by the rotating speed of the fan through a voltage transformer.

Both evaporators are of double-tube type in which the refrigerant flows in one direction through the inner tube while the heating medium flows in the opposite direction through the annular space between the inner and outer tubes. The heating medium of the high-temperature 


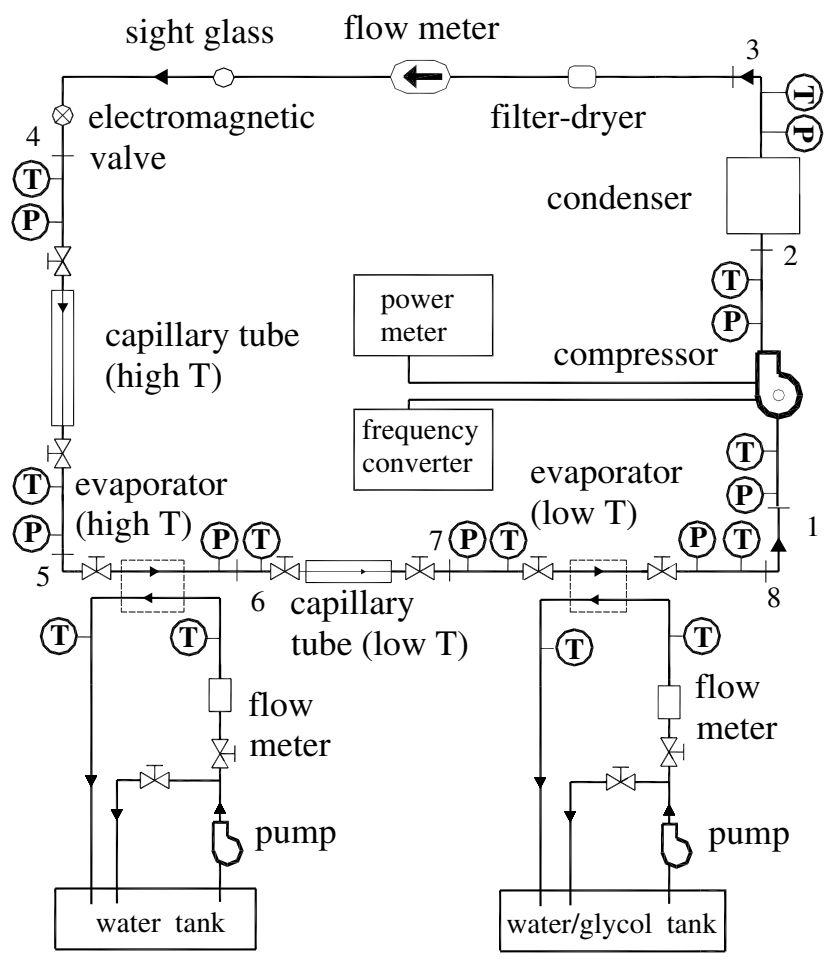

Fig. 1. Schematic diagram of the experimental system.

evaporator is water while that of low-temperature evaporator is water/glycol solution to avoid icing in the annular space between the tubes.

The selection of the capillary tubes involves some preliminary calculations and a trial-and-error test procedure for appropriate flow rates and operating temperatures. The cooling capacity of the low-temperature evaporator, $Q_{\mathrm{L}}$, may cover that of freezer of small to medium size. Note that the capillary tube for the high-temperature evaporator is not only smaller but also longer than that for the low-temperature counterpart. This situation is induced by the effect that part of the refrigerant flowing within the high-temperature capillary tube is in liquid state, while that within the low-temperature capillary tube is all in two-phase. The evaporators and the capillary tubes are all made of copper and are all insulated outside. The dimensions of the capillary tubes and the evaporators are listed in Table 1.

Table 1

Dimensions of capillary tubes and evaporators

\begin{tabular}{|c|c|c|c|c|c|c|c|}
\hline$d_{\mathrm{H}}(\mathrm{mm})$ & $d_{\mathrm{L}}(\mathrm{mm})$ & $L_{\mathrm{e}}(\mathrm{m})$ & $D_{\mathrm{i}, \mathrm{i}}(\mathrm{mm})$ & $D_{\mathrm{o}, \mathrm{i}}(\mathrm{mm})$ & $D_{\mathrm{i}, \mathrm{o}}(\mathrm{mm})$ & $D_{\mathrm{o}, \mathrm{o}}(\mathrm{mm})$ & \\
\hline 1.1 & 1.4 & 2 & 8.7 & 9.525 & 14.85 & 15.875 & \\
\hline$L_{\mathrm{H}}(\mathrm{m})$ & & & & $L_{\mathrm{L}}(\mathrm{m})$ & & & \\
\hline 1.5 & 1.75 & 2 & 2.25 & 0.2 & 0.4 & 0.6 & 0.8 \\
\hline
\end{tabular}


Both the heat-exchange loops are composed of a pump, a flow meter, and an electrically heated unit within the tank. Depending on the operation conditions, the refrigerant within the hightemperature evaporator is controlled between 15 and $20^{\circ} \mathrm{C}$, while that within the low-temperature evaporator is below $-10{ }^{\circ} \mathrm{C}$.

T-type thermocouples with monitor (APL7000\&S.B767, Jenco) with accuracy $\pm 0.1{ }^{\circ} \mathrm{C}$ and analogical pressure meters (316 SS TUBE\&SOCKET, Imperial) with accuracy $\pm 1 \%$ of the reading are used. Both the temperatures and pressures of the refrigerant before and after the compressor, both capillary tubes, and both evaporators are monitored. In addition, the temperatures of the water and the water/glycol solution of the heat-exchange loops are also recorded. The flow rates of the refrigerant, water, and water/glycol solution are measured with flow meters. The accuracy of the refrigerant flow meter (102-5T, Macmillan) is $\pm 3 \%$ in the range of the reading.

The controlled variables are the condensing pressure, the frequency of compressor, and the lengths of both capillary tubes, while the flow rates of both heating media are kept constant.

\section{Results and discussion}

\subsection{Pressure-enthalpy diagram}

Based on some test data and the thermodynamic properties of the refrigerant [11] and the heating media, the state of the refrigerant at different locations along the system is shown in Fig. 2,

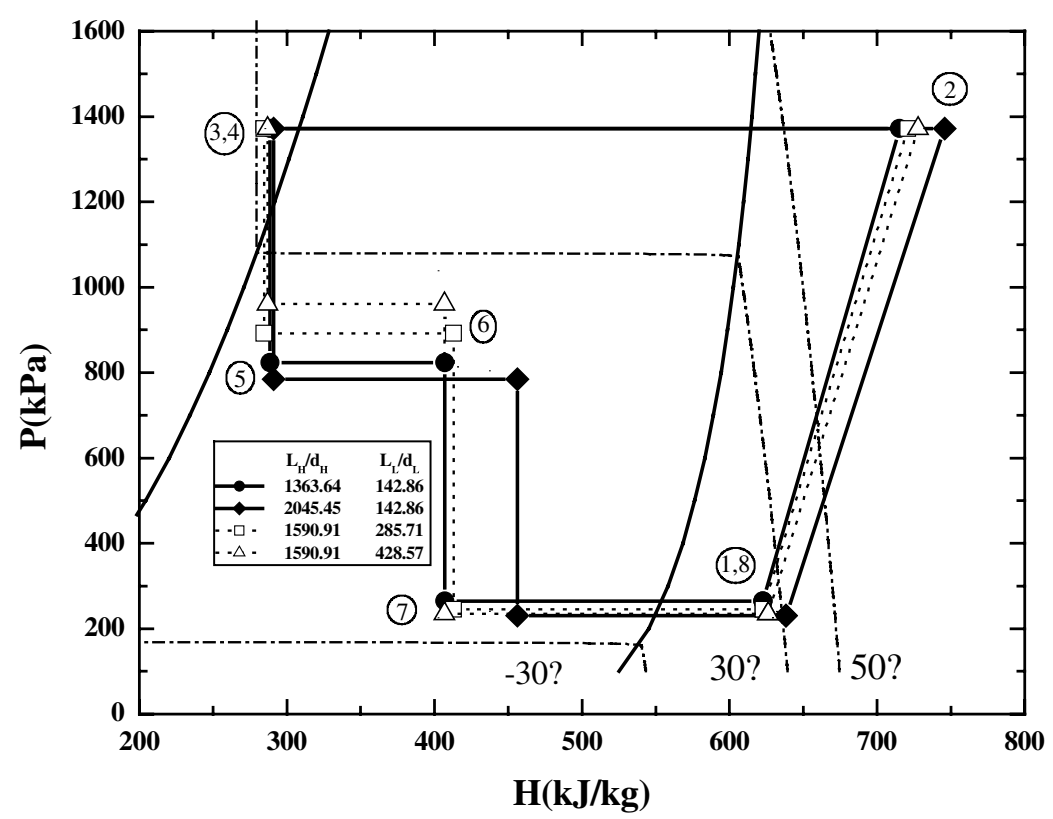

Fig. 2. $\mathrm{P}-\mathrm{h}$ diagram of the refrigerant within the system and the effect of lengths of capillary tubes on states of refrigerant $\left(P_{\mathrm{c}}=1372 \mathrm{kPa}\right)$. 
in which the encircled numbers indicate the corresponding locations as shown in Fig. 1. The details of Fig. 2 will be discussed later. To determine the states 6 and 7, the following equations for heat transfer are adopted:

$$
\begin{aligned}
& Q_{\mathrm{r}}=M_{\mathrm{r}} \cdot\left(H_{\mathrm{r}, \text { out }}-H_{\mathrm{r}, \text { in }}\right) \\
& Q_{\mathrm{hm}}=M_{\mathrm{hm}} \cdot c_{\mathrm{hm}} \cdot\left(T_{\mathrm{hm}, \text { in }}-T_{\mathrm{hm}, \text { out }}\right)
\end{aligned}
$$

Eq. (1) gives the actual heat absorbed by the refrigerant. As both evaporators are insulated, $Q_{\mathrm{r}}$ is taken as equal $Q_{\mathrm{hm}} . H_{\mathrm{r}, \text { in }}$ within the high-temperature evaporator, i.e. $H_{5}$, equals $H_{4}$ which is achievable through the measured $P_{4}$ and $T_{4}$. $H_{\mathrm{r} \text {,out }}$ within the high-temperature evaporator, i.e. $H_{6}$, can thus be obtained through Eq. (1). The location of point 6 can then be fixed by $H_{6}$ and the measured $P_{6}$. Knowing state 6, state 7 can be allocated by the measured $P_{7}$ and the fact that $H_{7}=H_{6}$. Similar situations occur for all other running conditions in the study.

\subsection{Effect of condensing pressure}

Fig. 3 shows the variation of the mass flow rate of the refrigerant $\left(M_{\mathrm{r}}\right)$ and the suction pressure $\left(P_{\text {suc }}\right)$ with the condensing pressure $\left(P_{\mathrm{c}}\right)$. It can be seen that both $M_{\mathrm{r}}$ and $P_{\text {suc }}$ increase with $P_{\mathrm{c}}$ for different length of high-temperature capillary tube, $L_{\mathrm{H}}$. For a given $L_{\mathrm{H}}$, the total pressure drop available for the refrigerant flowing through the system increases with $P_{\mathrm{c}}$. However, the pressure ratio across the compressor, $P_{\mathrm{c}} / P_{\text {suc }}$, varies with $M_{\mathrm{r}}$, which is a characteristic of the reciprocating compressor. Therefore $P_{\text {suc }}$ increases with $P_{\mathrm{c}}$. On the other hand, for a given $P_{\mathrm{c}}, M_{\mathrm{r}}$ decreases with $L_{\mathrm{H}}$ since more pressure drop is required for a longer capillary tube.

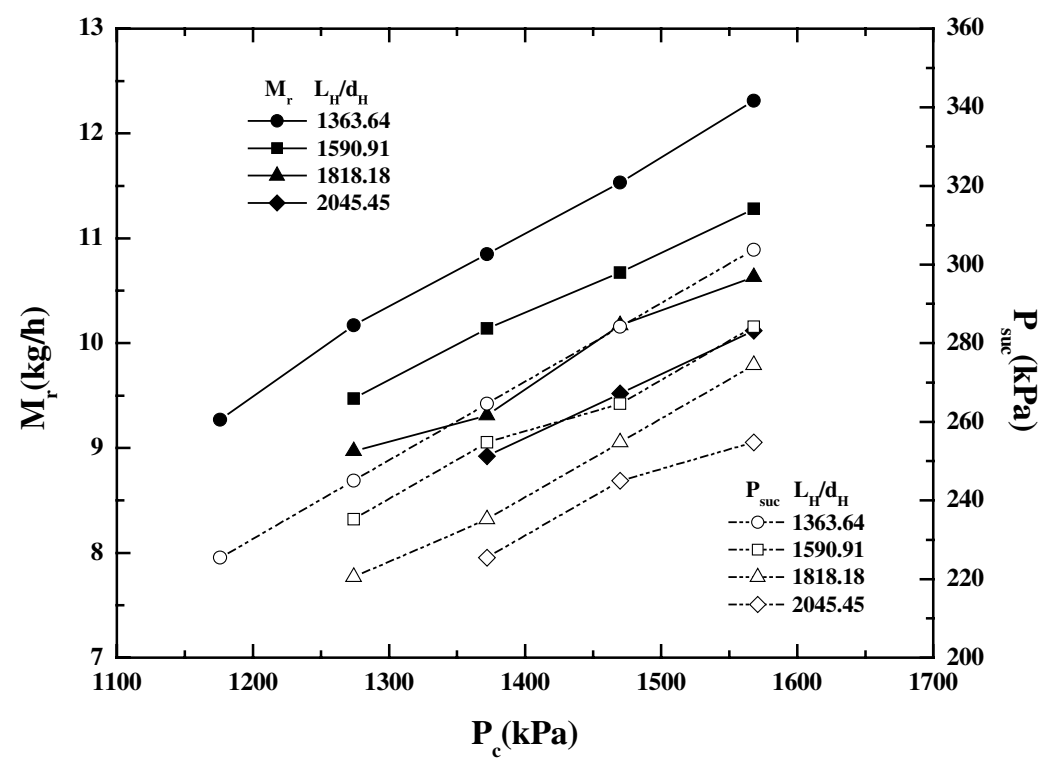

Fig. 3. Effects of condensing pressure on mass flow rate of refrigerant and suction pressure $\left(L_{\mathrm{L}} / d_{\mathrm{L}}=142.86\right)$. 


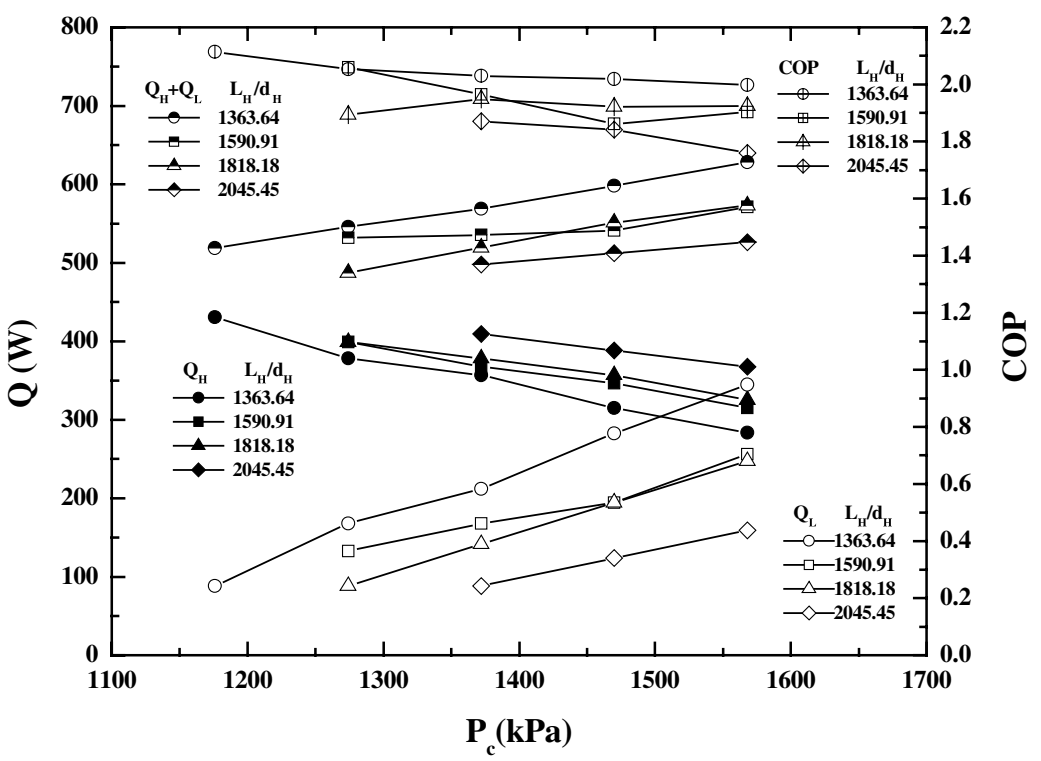

Fig. 4. Effects of condensing pressure on cooling capacities and COP $\left(L_{\mathrm{L}} / d_{\mathrm{L}}=142.86\right)$.

The effect of $P_{\mathrm{c}}$ on the cooling capacity of the two evaporators and COP is shown in Fig. 4. The heat-exchange rate between the working media within an evaporator may be obtained with Eqs. (1) and (2). However, it may also be expressed as

$$
Q=(U \cdot A) \cdot(\mathrm{LMTD})
$$

where the products of the overall heat transfer coefficients and the heat transfer areas and LMTD are defined as

$$
\begin{aligned}
& (U \cdot A)=\left(\frac{1}{h_{\mathrm{r}} \cdot A_{\mathrm{i}}}+R+\frac{1}{h_{\mathrm{hm}} \cdot A_{\mathrm{o}}}\right)^{-1} \\
& (\mathrm{LMTD})=\frac{\left(T_{\mathrm{hm}, \text { out }}-T_{\mathrm{r}, \text { in }}\right)-\left(T_{\mathrm{hm}, \text { in }}-T_{\mathrm{r}, \text { out }}\right)}{\ln \left(\frac{\left(T_{\mathrm{hm}, \text { out }}-T_{\mathrm{r}, \text { in }}\right)}{\left(T_{\mathrm{hm}, \text { in }}-T_{\mathrm{r}, \text { out }}\right)}\right)}
\end{aligned}
$$

$Q$ and LMTD in the high-temperature evaporator are taken as equal $Q_{\mathrm{H}}$ and $\mathrm{LMTD}_{\mathrm{H}}$, respectively, while those in the low-temperature evaporator are taken as equal $Q_{\mathrm{L}}$ and $\mathrm{LMTD}_{\mathrm{L}} \cdot T_{\mathrm{r}, \text { in }}$ in the high- and low-temperature evaporators are the saturation temperatures of the refrigerant corresponding to the measured $P_{5}$ and $P_{7}$, respectively, while $T_{\mathrm{r}, \text { out }}$ in the low-temperature evaporator is measured directly.

In the study, the conduction resistance, $R$, is fixed. In addition, the heat transfer coefficients of the heating media, $h_{\mathrm{hm}}$, is more or less constant since the flow rates of the heating media are kept constant and the variation in the temperatures of the heating media entering the evaporator is negligible. It is clear that both $Q_{\mathrm{H}}$ and $Q_{\mathrm{L}}$ are affected by LMTD and the heat coefficient of the refrigerant, $h_{\mathrm{r}}$, which is dominated by $M_{\mathrm{r}}$. 
Note that the average quality of the refrigerant in the high-temperature evaporator is lower than that in the low-temperature counterpart as shown in Fig. 2. With the criteria suggested by Shin et al. [12], the phase change in the high-temperature evaporator is thus mainly in the nucleate boiling region, while that in the low-temperature evaporator is mainly in the film boiling region. It has been pointed out that for nucleate boiling $h_{\mathrm{r}}$ does not change with $M_{\mathrm{r}}$, while for film boiling, $h_{\mathrm{r}}$ increases with $M_{\mathrm{r}}$ [12]. $Q_{\mathrm{H}}$ is, therefore, dominated completely by $\mathrm{LMTD}_{\mathrm{H}}$, while $Q_{\mathrm{L}}$ is affected by $h_{\mathrm{r}, \mathrm{L}}$ along with $\mathrm{LMTD}_{\mathrm{L}}$.

With $L_{\mathrm{H}}$ and $L_{\mathrm{L}}$ fixed, the refrigerant enters each evaporator with higher pressure when $P_{\mathrm{c}}$ is increased. Therefore, both $\mathrm{LMTD}_{\mathrm{H}}$ and $\mathrm{LMTD}_{\mathrm{L}}$ decrease with increasing $P_{\mathrm{c}}$. The decrease of $Q_{\mathrm{H}}$ with $P_{\mathrm{c}}$ shown in Fig. 4 is then induced entirely by the decreasing $\mathrm{LMTD}_{\mathrm{H}}$. On the other hand, the increase of $Q_{\mathrm{L}}$ with $P_{\mathrm{c}}$ indicates that the effect of $h_{\mathrm{r}, \mathrm{L}}$ dominates that of $\mathrm{LMTD}_{\mathrm{L}}$. It is noticeable that the total cooling capacity of the two evaporators, $Q_{\mathrm{t}}$, increases with $P_{\mathrm{c}}$ as shown in Fig. 4.

The variation of COP with $P_{\mathrm{c}}$ is shown in Fig. 4. COP is defined as

$$
\mathrm{COP}=\frac{Q_{\mathrm{t}}}{W_{\mathrm{r}}}
$$

where

$$
W_{\mathrm{r}}=M_{\mathrm{r}} \cdot \Delta H_{12}=M_{\mathrm{r}} \cdot\left(H_{2}-H_{1}\right)
$$

The specific enthalpies, $H_{1}$ and $H_{2}$, are determined through the states of the refrigerant just before and after the compressor, i.e. states $\left(P_{1}, T_{1}\right)$ and $\left(P_{2}, T_{2}\right)$. Note that since the study is concentrated on the effect of two evaporators with individual capillary tubes, isentropic compression is assumed in obtaining $H_{2}$. The increases of $M_{\mathrm{r}}, Q_{\mathrm{t}}$ and $W_{\mathrm{r}}$ with $P_{\mathrm{c}}$ are shown in Figs. 3-5, respectively. The net effect of $Q_{\mathrm{t}}$ and $W_{\mathrm{r}}$ makes COP of the system also decrease slightly with $P_{\mathrm{c}}$.

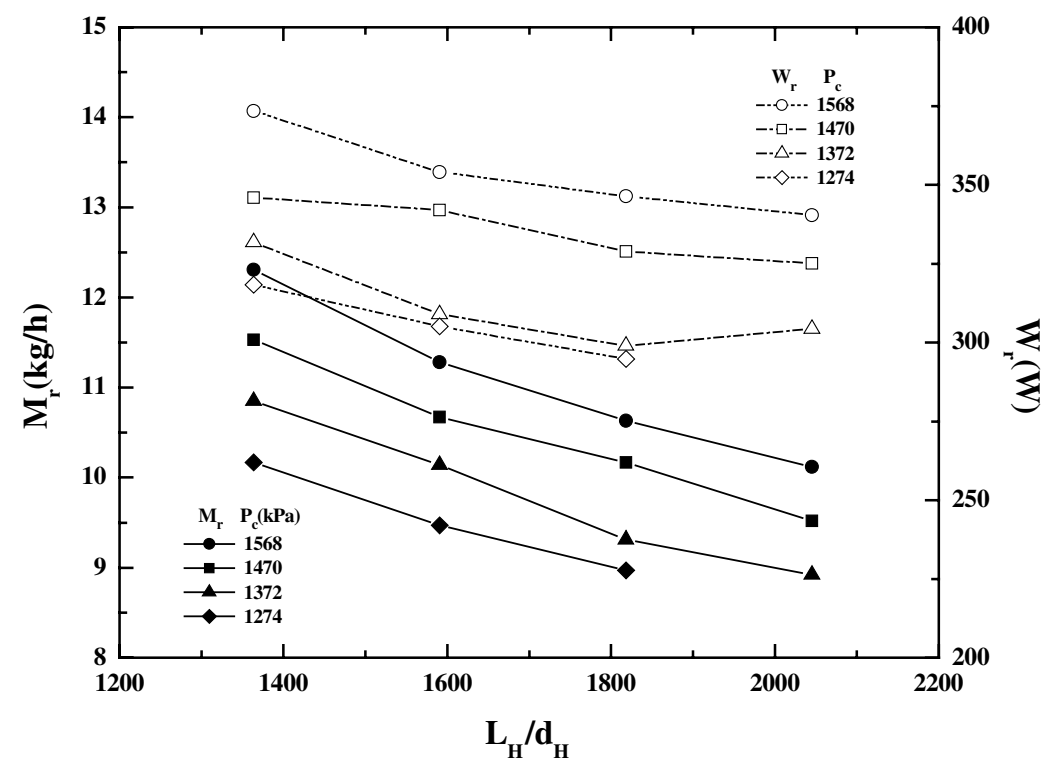

Fig. 5. Effects of length of capillary tube for high-temperature evaporator on mass flow rate of refrigerant and compression work $\left(L_{\mathrm{L}} / d_{\mathrm{L}}=142.86\right)$. 


\subsection{Effect of the length of capillary tubes}

The physical properties of the refrigerant at the inlet of the high-temperature capillary tube are more or less the same for different lengths of capillary tubes as shown in Fig. 2. $M_{\mathrm{r}}$ is therefore affected mainly by the length of capillary tube. Fig. 5 shows that for a given $P_{\mathrm{c}}$, both $M_{\mathrm{r}}$ and $W_{\mathrm{r}}$ decrease with increasing $L_{\mathrm{H}}$. Similar variation in $M_{\mathrm{r}}$ versus $L_{\mathrm{L}}$ can be found in Fig. 6. However, $W_{\mathrm{r}}$ seems to increase with $L_{\mathrm{L}}$ at high $P_{\mathrm{c}}$. The increase of $M_{\mathrm{r}}$ versus $P_{\mathrm{c}}$ is discussed in the previous subsection. For the case of high-temperature capillary tube, higher frictional loss is expected for longer tube, so $M_{\mathrm{r}}$ decreases with $L_{\mathrm{H}}$. For the case of low-temperature capillary tube, a check has been made to see whether the flow is chocked with the criterion suggested by Pate and Tree [13]. The results indicate that indeed choke occurs within the low-temperature capillary tubes for all of the four different $P_{\mathrm{c}}$. With choke occurring at the exit of the tube, the flow speed of the refrigerant there is more or less fixed. Lower density of the refrigerant at the exit of the low-temperature capillary tube can thus be expected at lower $P_{\text {suc }}$ induced by increasing $L_{\mathrm{L}}$. Therefore, $M_{\mathrm{r}}$ decreases with $L_{\mathrm{L}}$. On the other hand, $\Delta H_{12}$ increases with $L_{\mathrm{H}}$. Since $W_{\mathrm{r}}$, is a product of $\Delta H_{12}$ and $M_{\mathrm{r}}$, its variation versus $L_{\mathrm{H}}$ is thus more gradual than that of $M_{\mathrm{r}}$. It can be seen from Fig. 6 that a similar phenomenon occurs for the variation of $W_{\mathrm{r}}$ versus $L_{\mathrm{L}}$.

For a given $P_{\mathrm{c}}, Q_{\mathrm{H}}$ increases, while $Q_{\mathrm{L}}$ decreases with $L_{\mathrm{H}}$ as shown in Fig. 7. $Q_{\mathrm{H}}$ decreases and $Q_{\mathrm{L}}$ increases with $L_{\mathrm{L}}$ as shown in Fig. 8. As explained earlier, $\mathrm{LMTD}_{\mathrm{H}}$ increases with $L_{\mathrm{H}}$ and $h_{\mathrm{r}, \mathrm{H}}$ is not affected by $M_{\mathrm{r}}$. On the other hand, $h_{\mathrm{r}, \mathrm{L}}$ decreases with decreasing $M_{\mathrm{r}}$. However, when $L_{\mathrm{H}}$ is increased, the increasing $\mathrm{LMTD}_{\mathrm{L}}$ makes heat transfer higher. It seems that the effect of $h_{\mathrm{r}, \mathrm{L}}$ on $Q_{\mathrm{L}}$ is stronger than that of $\mathrm{LMTD}_{\mathrm{L}}$.

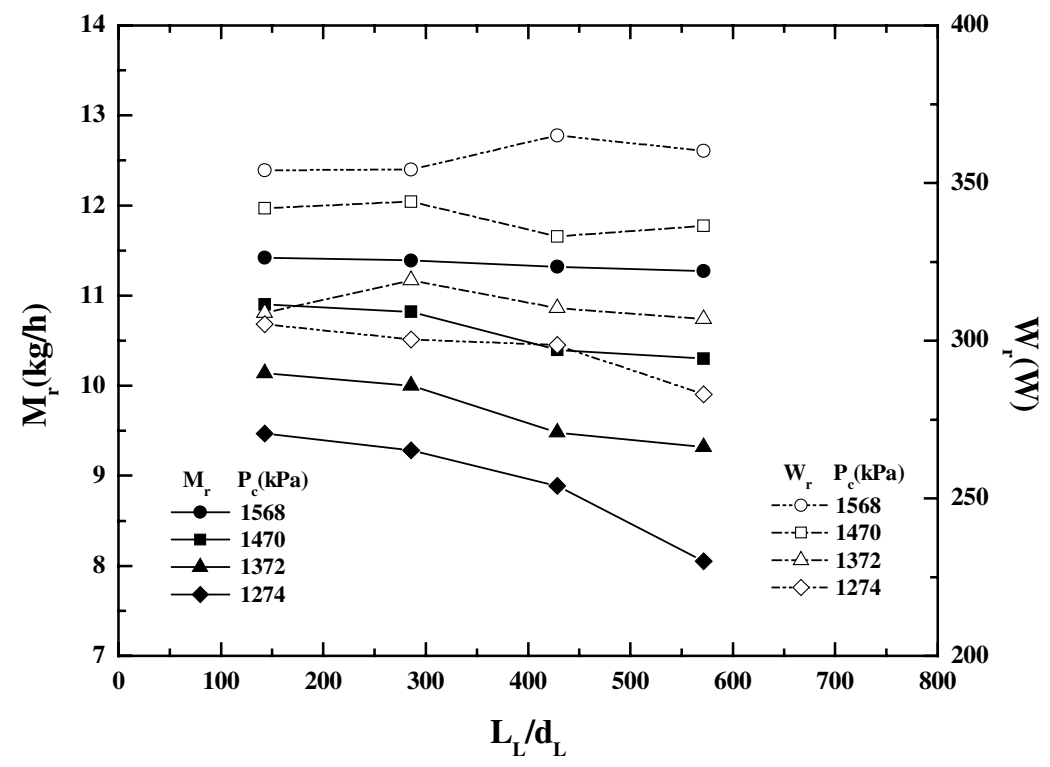

Fig. 6. Effects of length of capillary tube for low-temperature evaporator on mass flow rate of refrigerant and compression work $\left(L_{\mathrm{H}} / d_{\mathrm{H}}=1590.91\right)$. 


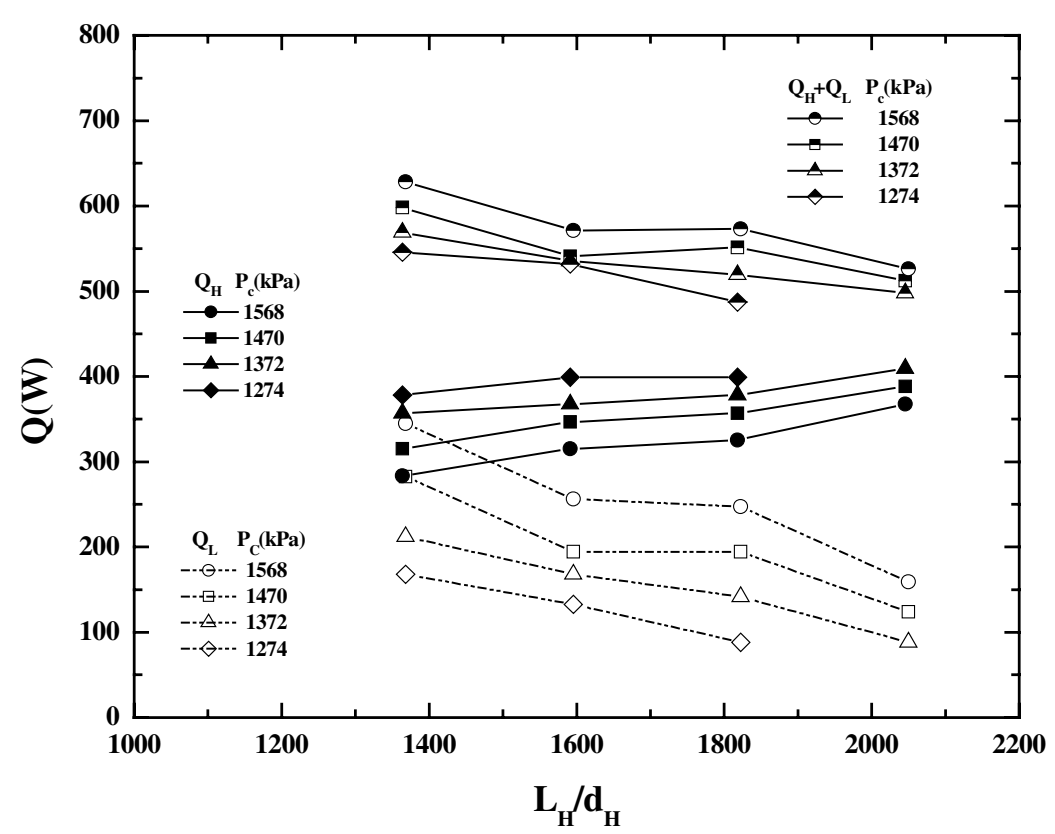

Fig. 7. Effects of length of capillary tube for high-temperature evaporator on cooling capacities $\left(L_{\mathrm{L}} / d_{\mathrm{L}}=142.86\right)$.

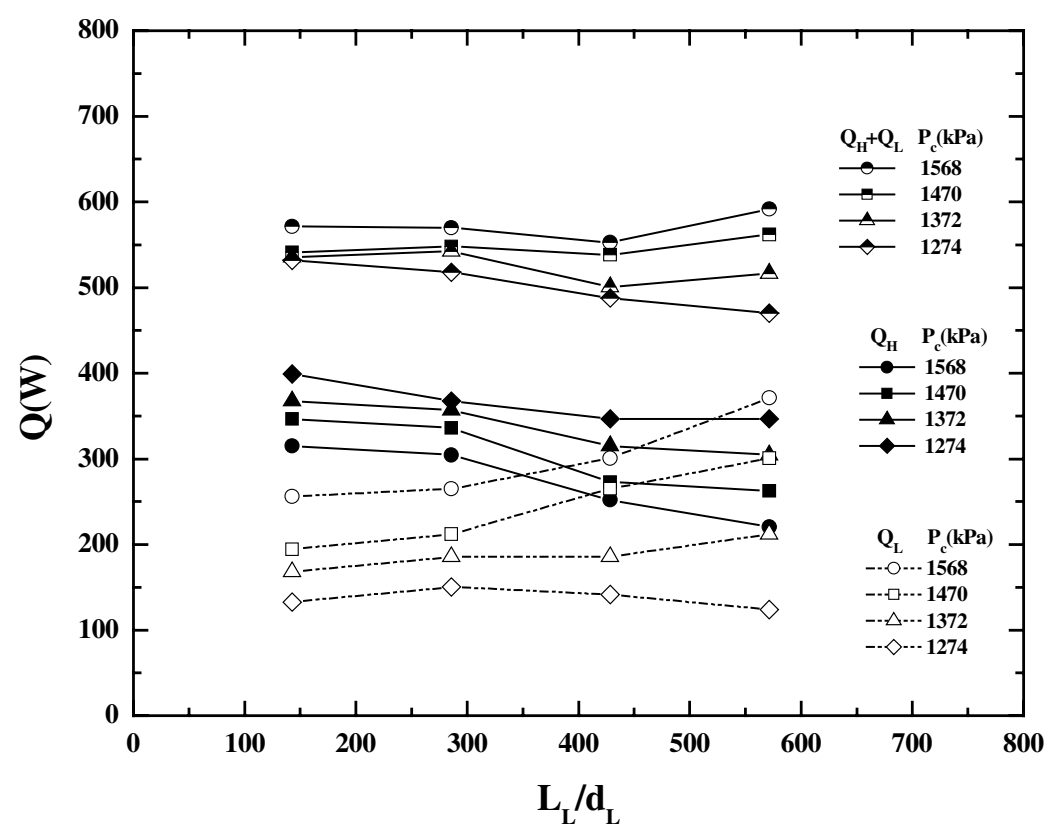

Fig. 8. Effects of length of capillary tube for low-temperature evaporator on cooling capacities $\left(L_{\mathrm{H}} / d_{\mathrm{H}}=1590.91\right)$.

For a given $L_{\mathrm{L}}$, the increasing $L_{\mathrm{H}}$ does not lower the pressure in the high-temperature evaporator, $P_{5}$ shown in Fig. 2. For an increase of $50 \%$ in $L_{\mathrm{H}}$ (from 1.5 to $2.25 \mathrm{~m}$ ), the pressure drop 
induced by the high-temperature capillary tube increases by $39.2 \mathrm{kPa}$, while that by the lowtemperature capillary tube decreases by $4.9 \mathrm{kPa}$. The relative changes of the pressure drops in comparing with their own original values within the high- and low-temperature capillary tubes are thus $+7.1 \%$ and $-0.9 \%$, respectively, while the total pressure difference between states 4 and 7 increases by only $3.1 \%$. The decreasing $M_{\mathrm{r}}$ with the increasing $L_{\mathrm{H}}$ may be the main reason for such a small increase in pressure drop. However, the effect of increasing $L_{\mathrm{L}}$ on the pressures of states 5 and 6 is more dramatic. With $L_{\mathrm{H}}$ fixed, an increase of $50 \%$ in $L_{\mathrm{L}}$ (from 0.4 to $0.6 \mathrm{~m}$ ) enlarges the pressure drop induced by the high- and low-temperature capillary tubes by $-14.3 \%$ and $12.1 \%$, respectively. However, the total pressure difference increases by only $0.9 \%$. It seems that the changes of the pressure difference in the individual capillary tubes induced by varying $L_{\mathrm{L}}$ are more than those by varying the high-temperature counterpart.

Although lengthening $L_{\mathrm{H}}$ does not induce much pressure drop between states 5 and 6 , it does bring about an apparent increase in the enthalpy of state 6 as shown in Fig. 2. Compared with the original enthalpy change in the high- and low-temperature evaporators, the increases induced by lengthening $L_{\mathrm{H}}$ by $50 \%$ are $39.5 \%$ and $-15.5 \%$, respectively, while those induced by lengthening $L_{\mathrm{L}}$ are $-6.9 \%$ and $4.7 \%$, respectively. The details of the variations may be worthy of further investigation.

\section{Conclusions}

With $P_{\mathrm{c}}, L_{\mathrm{H}}$ and $L_{\mathrm{L}}$ as the varying factors, the following conclusions may be drawn from the experimental study of the series-connected, two-evaporator refrigerating system with R-290 as the refrigerant.

1. Both $M_{\mathrm{r}}$ and $P_{\text {suc }}$ increase with $P_{\mathrm{c}}$ for fixed $L_{\mathrm{H}}$ and $L_{\mathrm{L}}$. In addition, $Q_{\mathrm{H}}$ decreases but $Q_{\mathrm{L}}$ increases with $P_{\mathrm{c}}$.

2. With other factors fixed, $Q_{\mathrm{H}}$ increases with $L_{\mathrm{H}}$, while $Q_{\mathrm{L}}$ and $M_{\mathrm{r}}$ decrease. On the other hand, $Q_{\mathrm{L}}$ increases with $L_{\mathrm{L}}$, while $Q_{\mathrm{H}}$ and $M_{\mathrm{r}}$ decrease.

3. $Q_{\mathrm{H}}$ and $Q_{\mathrm{L}}$ are mainly affected by the two effects: $h_{\mathrm{r}}$ and LMTD. For the case of the hightemperature evaporator, LMTD is the dominant factor. However, $h_{\mathrm{r}}$ also plays an important role in the case of the low-temperature evaporator.

4. The enthalpy changes of the refrigerant within the evaporators are strongly affected by $L_{\mathrm{H}}$. However, the evaporating pressures within the evaporators are influenced mainly by $L_{\mathrm{L}}$.

The variations of $M_{\mathrm{r}}, W_{\mathrm{r}}, Q_{\mathrm{H}}, Q_{\mathrm{L}}$ and COP versus $L_{\mathrm{H}}, L_{\mathrm{L}}$ and $P_{\mathrm{c}}$ studied in this work may be applied to the design of a two-evaporator refrigerating system. However, the operating ranges of $P_{\mathrm{c}}$ and the dimensions of the capillary tubes may be enlarged for clearer understanding of the effect of these factors. In addition, works on other environment-friendly refrigerants or the blends of them are also worthy of further investigation.

\section{References}

[1] M.S. Kim, W.J. Mulroy, D.A. Didion, Performance evaluation of two azeotropic refrigerant mixtures of HFC-134a with R-290 (Propane) and R-600a (Isobutane), J. Energy Resour. Technol., Trans. ASME 116 (1994) 148-154. 
[2] D. Jung, C.B. Kim, B.H. Lim, H.W. Lee, Testing of a hydrocarbon mixture in domestic refrigerators, ASHRAE Trans.: Symposia 102 (1) (1996) 1077-1084.

[3] M.A. Alsaad, M.A. Hammad, The application of propane/butane mixtures for domestic refrigerators, Appl. Thermal Eng. 18 (1998) 911-918.

[4] Y. Zhao, M. Yitai, L. Yie, C. Zhonghai, M. Lishan, The performance of some substitutes for HCFC22 under varying operating conditions, Appl. Thermal Eng. 19 (1999) 801-806.

[5] B. Tashtoush, M. Tahat, M.A. Shudeifat, Experimental study of new refrigerant mixtures to replace R12 in domestic refrigerators, Appl. Thermal Eng. 22 (2002) 495-506.

[6] A. Lorenz, K. Meutzner, On application of nonazeotropic two-component refrigerants in domestic refrigerators and home freezers, in: XIV International Congress of Refrigeration, Moscow, 1975.

[7] R.J. Rose, D.S. Jung, R. Radermacher, Testing of domestic two-evaporator refrigerators with zeotropic refrigerant mixtures, ASHRAE Trans. 98 (1992) 216-226.

[8] D.S. Jung, R. Radermacher, Performance simulation of a two-evaporator refrigerator-freezer charged with pure and mixed refrigerants, Int. J. Refrig. 14 (1991) 254-263.

[9] K.E. Simmons, K. Kim, R. Radermacher, Experimental study of independent temperature control of refrigerator compartments in a modified Lorenz-Meutzner cycle, ASME AES 34 (1995) 67-73.

[10] K.E. Simmons, I. Haider, R. Radermacher, Independent compartment temperature control of Lorenz-Meutzner and modified Lorenz-Meutzner cycle refrigerators, ASHRAE Trans. 102 (1) (1996) 1085-1092.

[11] M.O. McLinden, E.W. Lemmon, S.A. Klein, A.P. Peskin, NIST thermodynamic properties of refrigerants and refrigerant mixtures database (REFPROP). US Department of Commerce, National Institute of Standards and Technology, Gaithersburg, MD, 6.0, 1998.

[12] J.Y. Shin, M.S. Kim, S.T. Ro, Experimental study on forced convective boiling heat transfer of pure refrigerants and refrigerant mixtures in a horizontal tube, Int. J. Refrig. 20 (4) (1997) 267-275.

[13] M.B. Pate, D.R. Tree, An analysis of choked flow conditions in a capillary tube-suction line heat exchanger, ASHRAE Trans. 93 (1) (1987) 368-380. 